\title{
EFECTOS DE LA ENDOGAMIA SOBRE PARÁMETROS REPRODUCTIVOS EN VACAS HOLSTEIN Y JERSEY DE COSTA RICA ${ }^{1}$
}

\author{
Judyana Aguirre-Valverde ${ }^{2}$, Bernardo Vargas-Leitón ${ }^{2}$, Juan José Romero-Zúñiga ${ }^{2}$
}

\begin{abstract}
RESUMEN
Efectos de la endogamia sobre parámetros reproductivos en vacas Holstein y Jersey de Costa Rica. El objetivo de este trabajo fue analizar el efecto de los niveles de consanguinidad (F) en vacas Holstein y Jersey nacidas entre enero de 1995 y diciembre de 2010 en hatos lecheros especializados de Costa Rica. Los datos de la edad a primer parto (EPP), días abiertos (DA) y servicios por concepción (SPC) se procesaron en el año 2011 mediante estadística descriptiva, regresión lineal múltiple (modelo mixto) y regresión logística. Se empleó la base de datos $(n=263$ 804) del programa VAMPP Bovino de la Escuela de Medicina Veterinaria de la Universidad Nacional, Heredia, Costa Rica. Se utilizó como punto de corte un valor $\mathrm{F} \geq 6,25 \%$ (alto/ riesgo). Resultó un 17,9\% de animales con endogamia con $\mathrm{F}$ promedio de $0,42 \%(0,49 \%$ y $0,55 \%$ para Holstein y Jersey). Hubo 22,6\% de Holstein consanguíneos y 20,3\% de Jersey; sin embargo, en Jersey hubo animales con $\mathrm{F}$ más elevados. Las vacas con endogamia tuvieron un promedio de $\mathrm{F}$ de $2,16 \%$ para Holstein y $2,98 \%$ para Jersey. Las vacas con $\mathrm{F}$ bajos tuvieron 18 días menos de $\operatorname{EPP}(\mathrm{p}<0,01)$, en relación a las que tienen $\mathrm{F}$ alto. En DA hubo 0,53 días más para animales con $\mathrm{F}$ bajo $(\mathrm{p}=0,71)$, mientras que en SPC hubo 0,08 más servicios en vacas con $\mathrm{F}$ bajo $(\mathrm{P}<0,01)$. No hubo riesgo de presentar DA o SPC mayores en animales $\mathrm{F} \geq 6,25 \%$, lo que sí ocurrió para la EPP $(\mathrm{OR}=1,23$; IC95\% 1,10-1,38). La endogamia no afectó los DA, pero sí levemente los SPC y la EPP, en vacas Holstein y Jersey.
\end{abstract}

Palabras clave: consanguinidad en vacas, edad al primer parto, servicios por concepción, días abiertos, ganado lechero.

\begin{abstract}
Effects of inbreeding on reproductive parameters of Costa Rican Holstein and Jersey cows. The objective of this study was to analyze the effect of high inbreeding (F) levels $(\geq 6.25 \%)$ on reproductive parameters of Holstein and Jersey cows born between Jan 1995 and Dec 2010 in specialized dairy herds in Costa Rica. Data on age at first calving (AFC), open days (DO) and services per conception (SPC) from $(n=263$ 804) from the VAMPP national data base, at the Veterinary School of the Universidad Nacional, was used. An $\mathrm{F}$ value $\geq 6.25 \%$ (high/risk) was used as a cut-off point. The data were processed using descriptive statistics, multiple linear regression (mixed model), and logistic regression. A $17.9 \%$ of animals had some inbreeding, with an $\mathrm{F}$ average of $0.42 \%$ ( $0.49 \%$ and $0.55 \%$ for Holstein and Jersey). $22.6 \%$ of Holstein and $20.3 \%$ of Jersey were inbreed; however, in Jersey, there were cows with higher F. Cows with some inbreeding have an average $\mathrm{F}$ of $2.16 \%$ for Holstein and $2.98 \%$ for Jersey. Furthermore, cows with low F had $18 \mathrm{~d}$ less of AFC $(\mathrm{p}<0.01)$ compared to those with high F. Regarding the DO, there were $+0.53 \mathrm{~d}$ for animals with low $\mathrm{F}(\mathrm{P}=0.71)$; while the cows with low $\mathrm{F}$ had +0.08 SPC $(\mathrm{P}<0.01)$. There was no risk of DO or SPC in animals with $\mathrm{F} \geq 6.25 \%$; which occurred for AFC $(\mathrm{OR}=1.23$; 95\% CI: 1.10-1.38). According to this study, inbreeding did not affect the DO, but slightly affects the SPC and the AFC in Holstein and Jersey cows.
\end{abstract}

Keywords: inbreeding, age at first calving, services per conception, open days, dairy cattle.

\footnotetext{
1 Recibido: 12 de febrero, 2013. Aceptado: 28 de octubre, 2013. Parte de la tesis de la primer autora, para optar por su título de Magister Scientiae en Producción Animal Sostenible por el Posgrado en Ciencias Veterinarias Tropicales de la Escuela de Medicina Veterinaria de la Universidad Nacional, Costa Rica.

2 Producción Animal Sostenible, Posgrado en Ciencias Veterinarias Tropicales y Programa de Investigación en Medicina Poblacional, Escuela de Medicina Veterinaria. Universidad Nacional. Costa Rica. Apdo. Postal: 304-3000 Heredia. Teléfono: (506) 25624566. judyveterinaria@ yahoo.es, bernardo.vargas.leiton@una.cr, juan.romero.zuniga@una.cr (Autor para correspondencia).
} 


\section{INTRODUCCIÓN}

Con el fin de mejorar la eficiencia productiva y reproductiva de un hato bovino, principalmente uno de lechería, se utilizan técnicas reproductivas especializadas como la inseminación artificial (IA), que permite incrementar el progreso genético mediante aumento en la intensidad de selección y reducción del intervalo entre generaciones. Estas técnicas generalmente inducen a una disminución de la cantidad de toros utilizados con fines reproductivos, a los que se extrae su semen y que se usan con frecuencia para aprovechar sus características positivas pero sin ningún control en cada explotación (Vargas y Gamboa 2008). Adicionalmente, las compañías fabricantes de pajillas de semen para IA, a menudo, utilizan hijos superiores de algunos de sus mejores toros, produciendo así varias generaciones de toros emparentados entre ellos, que al ser utilizados en las fincas, durante varios años, podría traer como efecto colateral gran cantidad de individuos emparentados entre sí (endogamia). En estos animales se reporta, con alta frecuencia, una depresión en sus caracteres productivos y reproductivos. Investigaciones realizadas a nivel mundial (Caraviello 2004b, McParland et al. 2007, Vargas y Gamboa 2008), comprobaron que esta depresión está presente, en mayor o menor grado, en los hatos lecheros especializados en los que no hay un programa de control reproductivo definido.

Los aspectos más importantes en los que está implicada la endogamia en reproducción son: aumento de la incidencia de distocia en vacas (Smith et al. 1998, Adamec et al. 2006), retención de membranas fetales (Romero et al. 2007), servicios por concepción, días abiertos (Nicholas 2003, Caravaca et al. 2005, RuizFlores et al. 2006), disminución en fertilidad (Caraviello 2004a, McParland et al. 2009), reducción en la libido, aumentos de la esterilidad gamética, mortalidad embrionaria (Lazzari et al. 2011) y edad al primer parto (Smith et al. 1998). Las alteraciones encontradas por cada $1 \%$ de incremento de consanguinidad son: $+0,55$ (Smith et al. 1998), +0,5 (Casas y Tewolde 2001) y +0,48 (Røkouei et al. 2010) meses de edad al primer parto, y entre $+0,73 \mathrm{y}+0,53$ meses en el intervalo entre partos (Røkouei et al. 2010, Mc Parland et al. 2007), así como $0,46 \%$ de probabilidad de distocia (Smith et al. 1998).

Esta investigación tiene como objetivo analizar el efecto de los niveles de consanguinidad en vacas
Holstein y Jersey en hatos lecheros especializados de Costa Rica.

\section{MATERIALES Y MÉTODOS}

\section{Fuente de datos}

Se realizó un estudio en el año 2011 longitudinal retrospectivo con los registros de 1395 fincas lecheras de Costa Rica. Esta información pertenece a la base de datos nacional del programa VAMPP Bovino (Veterinary Automated Management and Production Control Programme; Noordhuizen y Buurman 1984), en el Centro Regional de Informática para la Producción Animal Sostenible (CRIPAS), de la Escuela de Medicina Veterinaria - Universidad Nacional (EMV-UNA). De estos datos, se seleccionaron las vacas que nacieron en el periodo comprendido entre el $1^{\circ}$ de enero de 1995 y el 30 de diciembre de 2010, y que tuvieran datos de genealogía que permitieran el cálculo de su coeficiente de consanguinidad (F). En total, 545 hatos lecheros cumplieron con los criterios de inclusión del estudio.

\section{Método de recolección de datos}

Diariamente, en cada finca, los productores anotan en una libreta de apuntes los datos de los eventos que ocurren. Luego, esta información es introducida en el programa VAMPP Bovino por ellos mismos o por los técnicos que trabajan brindando asistencia en la granja. El personal del proyecto CRIPAS recolecta y centraliza, al menos dos veces al año, la información contenida en el programa VAMPP Bovino de cada finca en forma descentralizada (Romero 2005). Hoy día, VAMPP Bovino es utilizado por alrededor de 1500 granjas lecheras especializadas en Costa Rica, algunas con información de hace 30 años.

\section{Descripción de las variables}

La variable independiente principal es el coeficiente de endogamia $(F)$ de cada vaca, siendo las variables dependientes: edad al primer parto (EPP), servicios por concepción (S/C) y días abiertos (DA). Se tomaron en consideración otras covariables y fuentes de variación: raza, número de lactancias, zona ecológica (ecozona), 
hato, año y época de parto. Estas tres últimas fueron combinadas en una sola variable que se constituyó en la de efecto aleatorio. Las variables dependientes e independientes, así como la forma en que se utilizaron, se describen en el Cuadro 1.

\section{Edición y análisis de datos}

Los animales nacidos en años anteriores a 1985 o después de 2008, se tomaron como nacidos en esos años. Si el año de parto era mayor a 2010 , se reubicaron en ese año; esto debido al bajo volumen de datos para fechas de nacimiento o de parto para esos años utilizados como límites.

Para determinar el efecto de la endogamia sobre los parámetros reproductivos, se incluyeron en el estudio únicamente las vacas Holstein y Jersey; asimismo, los cruces con $75 \%$ o más de la raza fueron considerados como "puras". Las otras razas puras, como Guernsey y Pardo Suizo, fueron tomadas en cuenta solo en la fase de estadística descriptiva.

Posteriormente, se eliminaron los animales que no poseían ningún grado de consanguinidad; asimismo, los registros con más de ocho $\mathrm{S} / \mathrm{C}$ se asumieron como ocho. De igual manera, la EPP mayor a los $60 \mathrm{~m}$ se trasladó a $60 \mathrm{~m}$ y los valores del intervalo parto concepción menores a 20 d y mayores a 450 d, se ubicaron en esos límites.

En cuanto a la variable zonas de vida según Holdridge (1987) se eliminaron tres debido a que hubo pocos datos y que no aportaban mucha información al estudio: bosque muy húmedo montano (bmh-M), bosque pluvial premontano (bp-PM) y bosque seco tropical (bs-T) (Cuadro 2).

Cuadro 1. Descripción de las variables reproductivas utilizadas en el análisis de la situación de la endogamia en fincas lecheras de Costa Rica. Período 1995 a 2010.

\begin{tabular}{|c|c|c|c|c|}
\hline Variable & Condición & Tipo & Nivel & Descripción \\
\hline \multirow[t]{3}{*}{ Días abiertos } & Dependiente & Continua & & Días \\
\hline & & Discreta & Bajo & $<140$ \\
\hline & & & Alto & $\geq 140$ \\
\hline \multirow[t]{3}{*}{ Servicios/concepción } & Dependiente & Continua & & \\
\hline & & Discreta & Bajo & $<1,5$ \\
\hline & & & Alto & $\geq 1,5$ \\
\hline \multirow[t]{3}{*}{ Edad al primer parto } & Dependiente & Continua & & Meses \\
\hline & & Discreta & Bajo & $<28$ \\
\hline & & & Alto & $\geq 28$ \\
\hline \multirow[t]{3}{*}{ Endogamia } & Independiente & Continua & & \\
\hline & & Discreta & Bajo & $<6,25 \%$ \\
\hline & & & Alto & $\geq 6,25 \%$ \\
\hline \multirow[t]{2}{*}{ Raza } & Independiente & Discreta & Holstein & $\geq 6 / 8$ Holstein \\
\hline & & & Jersey & $\geq 6 / 8$ Jersey \\
\hline \multirow[t]{6}{*}{ Número de lactancia } & Independiente & Continua & & \\
\hline & & Discreta & 1 & Primerizas \\
\hline & & & 2 & Segunda \\
\hline & & & 3 & Tercera \\
\hline & & & 4 & Cuarta \\
\hline & & & 5 & 5 o más \\
\hline Ecozonas & Independiente & Discreta & 1 a 7 & Zonas de vida (Holdridge1987) \\
\hline Hato & Independiente & Discreta & & Base de datos VAMPP \\
\hline Año de parto/nacimiento & Independiente & Discreta & 1994-2009 & Período del estudio \\
\hline \multirow{2}{*}{$\begin{array}{l}\text { Época de parto/ } \\
\text { nacimiento }\end{array}$} & Independiente & Discreta & Seca & Diciembre, enero-abril \\
\hline & & & Lluviosa & Mayo-noviembre \\
\hline
\end{tabular}


Cuadro 2. Rangos de variación en altitud, precipitación y temperatura en zonas agroecológicas de Costa Rica, con presencia de sistemas de producción lecheros ${ }^{1}$. Costa Rica. Período enero 1995 a diciembre 2010.

\begin{tabular}{lcccc}
\hline Zona agroecológica & Código & Altitud $(\mathbf{m s n m})$ & Precipitación (mms/año) & Temperatura $\left({ }^{\circ} \mathbf{C}\right)$ \\
\hline B. muy húmedo tropical & bmh-T & $0-500$ & $4000-8000$ & $>24$ \\
B. muy húmedo premontano & bmh-P & $500-1500$ & $2000-4000$ & $18-24$ \\
B. húmedo montano bajo & bh-MB & $1500-2000$ & $1000-2000$ & $12-17$ \\
B. pluvial montano bajo & bp-MB & $1500-2000$ & $4000-8000$ & $12-17$ \\
B. húmedo tropical & bh-T & $0-500$ & $2000-4000$ & $>24$ \\
B. húmedo premontano & bh-P & $500-1500$ & $1000-2000$ & $18-24$ \\
B. muy húmedo montano bajo & bmh-MB & $1500-2000$ & $2000-4000$ & $12-17$ \\
\hline
\end{tabular}

${ }^{1}$ Tomado de Vargas y Ulloa (2008). Desarrollado con base en Holdridge (1987).

\section{Procesamiento estadístico}

El procesamiento de datos se realizó mediante tres fases:

1. Estadística descriptiva, mediante medidas de frecuencia, de tendencia central y de dispersión. Fueron utilizados los procedimientos PROC UNIVARIATE, PROC FREQ y PROC MEANS del software SAS (2009).

2. Estimación del efecto absoluto mediante la regresión lineal múltiple utilizando un modelo mixto (PROC MIXED, SAS 2009). Se creó un modelo para cada variable dependiente. fue:

El modelo estadístico para la edad a primer parto

$Y_{i j k l m}=\mu 0+e n d o_{i}+h a n_{j}+e p_{k}+r a z a_{l}+e c o_{m}+e_{i j k l m}$

Donde:

$Y_{i j k l m}=$ Edad al primer parto (meses)

$\mu 0=$ media general de la variable de respuesta

endo ${ }_{i}=$ efecto fijo de la $i$-ésima endogamia $(i=$ $0 \ldots ;$ porcentaje de endogamia)

$h a n_{j}=$ efecto aleatorio del j-ésimo hato/año de nacimiento

$e p_{k}=$ efecto fijo de $k$-ésima época de nacimiento ( $k=0-1 ; 0=$ seca, $1=$ lluviosa)

$r a z a_{l}=$ efecto fijo de la $l$-ésima raza $(l=1-2 ; 1=$

Holstein, 2= Jersey) $1-7)$

eco ${ }_{m}=$ efecto fijo de la $m$-ésima ecozona $(m=$ $e_{i j k l m}=$ efecto aleatorio residual

El modelo estadístico para los servicios por concepción fue:

$$
\begin{aligned}
Y_{i j k l m n}= & \mu 0+e n d o_{i}+h a p_{j}+e p_{k}+\operatorname{raza}_{l}+\text { nlac }_{m}+e c o_{n} \\
& +e_{i j k l m n}
\end{aligned}
$$

Donde:

$Y_{i j k l m n}=$ servicios por concepción (número de servicios)*

$\mu 0=$ media general de la variable de respuesta

endo $_{i}=$ efecto fijo de la $i$-ésima endogamia $(i=$ $0 \ldots ;$ porcentaje de endogamia)

$h a p_{j}=$ efecto aleatorio del $j$-ésimo hato/año de parto $e p_{k}=$ efecto fijo de $k$-ésima época de parto $(k=$ $0-1 ; 0=$ seca, $1=$ lluviosa)

$r a z a_{l}=$ efecto fijo de la l-ésima raza $(l=1-2 ; 1=$ Holstein, 2= Jersey)

nlac $_{m}=$ efecto fijo de la $m$-ésima lactancia $(m=$ $1-5 ; 1=1,2=2,3=3,4=4,5=\geq 5$ lactancia)

eco ${ }_{n}=$ efecto fijo de la $n$-ésima ecozona $(n=1-7)$

$e_{i j k l m n}=$ efecto aleatorio residual

*Este modelo se repite para la variable dependiente días abiertos (número de días).

3. Estimación del riesgo (razón de posibilidades) de que una vaca con niveles altos ( $\geq 6,25 \%$ ) de endogamia (Florio 2005, Romero et al. 2007) presenten parámetros reproductivos desfavorables (DA, EPP y $\mathrm{S} / \mathrm{C}$ altos), según la raza y el número de lactancia. 
Esto se realizó mediante regresión logística utilizando el procedimiento Proc Logistic, SAS/STAT ${ }^{\circledR}$ ver 9.2. (SAS 2009).

\section{RESULTADOS Y DISCUSIÓN}

\section{Estadística descriptiva}

Se trabajó con un total de 541212 lactancias. Luego de la reducción y edición de los datos en que se seleccionaron las vacas, que tenían datos genealógicos con los que se pudo calcular el coeficiente de consanguinidad, quedaron 263805 registros. Resultó una tasa global de animales con algún grado de endogamia de 17,9\% ( $n=47221)$, que es ligeramente más elevado que el estudio de Hudson y Van Vleck (1984), que reportaron un valor de $16,89 \%$, pero que contrasta con el estudio de Romero et al. (2007), con una tasa de 6,04\%. Esta diferencia se puede deber a que el número de las fincas seleccionadas en el estudio de Romero et al. fue menor, así como una diferencia en el porcentaje de animales Jersey en ambos estudios, que fue mayor en el presente estudio. Además, Romero et al. (2007), incluyeron datos hasta $1996 \mathrm{y}$, en aquel momento, el programa VAMPP Bovino no contaba con una base de datos de toros tan completa como lo tiene actualmente, ni tenía el motor de búsqueda de ancestros comunes para la estimación del coeficiente de endogamia. Además, si bien tiene un peso menor en esta importante diferencia de tasas globales de endogamia, se ha observado un incremento gradual de esta en los hatos lecheros; por ejemplo, en los Estados Unidos de América, la tasa de variación interanual de $\mathrm{F}$ fue de 0,14 y 0,24 , para Holstein y Jersey, respectivamente, para el período comprendido entre 1994 y 2004 (AIPL 2005, USDA 2012).

Por otra parte, se presentó una diferencia en la EPP de $+1,64 \mathrm{~m}$ en Holstein, $+39 \mathrm{~d}$ de DA en las Jersey $\mathrm{y}+0,18 \mathrm{SPC}$ en las Holstein. El promedio general de SPC fue de 2,02, con valores que oscilaron entre $1 \mathrm{y}$ 16. Por otro lado, la EPP promedio fue de $30,23 \mathrm{~m}$, con un valor mínimo de 18 y un máximo de 48 ; asimismo, el promedio de DA fue de 140,9 d (Cuadro 3).

Casi la mitad de los animales en estudio fueron Holstein con un 58\%, el 37,5\% correspondió a Jersey, mientras el 3,2\% fue Pardo Suizo y el 1,3\% Guernsey; las otras razas solo representaron un $0,01 \%$.

\section{Niveles de endogamia}

La raza Holstein presentó 22,6\% y la Jersey $20,3 \%$ de vacas con algún grado de endogamia, resultados que son similares a los obtenidos por Vargas y Gamboa (2008) con 21,2\% para Holstein y $23,2 \%$ para Jersey en la misma población, con la diferencia de que en este estudio la de mayor porcentaje fue Holstein; sin embargo, la raza Jersey fue la que tuvo mayor porcentaje de vacas con grados de endogamia considerados de riesgo para obtener menor rendimiento productivo (Cuadro 4).

El promedio de consanguinidad, tomando los aparentes ceros para la Holstein y Jersey, fue de $0,49 \%$ y $0,55 \%$, valores que son más bajos que los

Cuadro 3. Promedios de indicadores reproductivos de la raza Holstein, Jersey y Pardo Suizo, con algún grado de consanguinidad, en vacas de fincas lecheras especializadas de Costa Rica. Período enero 1995 a diciembre 2010.

\begin{tabular}{|c|c|c|c|c|c|c|}
\hline \multirow[t]{2}{*}{ Variable } & \multicolumn{2}{|c|}{ Holstein } & \multicolumn{2}{|c|}{ Jersey } & \multicolumn{2}{|c|}{ Pardo Suizo } \\
\hline & $\mathbf{N}$ & Media & $\mathbf{N}$ & Media & $\mathbf{N}$ & Media \\
\hline Consanguinidad (\%) & 27365 & 2,16 & 17702 & 2,68 & 1502 & 2,60 \\
\hline $\operatorname{SPC}\left(\mathrm{N}^{\circ}\right)$ & 12309 & 2,09 & 7661 & 1,91 & 762 & 2,391 \\
\hline EPP (meses) & 14239 & 28,46 & 8497 & 26,82 & 882 & 27,68 \\
\hline DA (días) & 12309 & 159,69 & 7661 & 120,06 & 762 & 186,53 \\
\hline
\end{tabular}

$\mathrm{SPC}=$ servicios por concepción $\mathrm{EPP}=$ edad al primer parto; $\mathrm{DA}=$ días abiertos. $\mathrm{N}=$ tamaño de muestra. 
Cuadro 4. Resumen estadístico de endogamia en los animales en estudio y sus diferentes valores de nivel de riesgo, en vacas de fincas lecheras especializadas de Costa Rica. Período enero 1995 a diciembre 2010.

\begin{tabular}{lcccc}
\hline & \multicolumn{3}{c}{ Raza } \\
\cline { 2 - 4 } & & Holstein & Jersey & Pardo Suizo \\
\hline $\mathrm{N}$ & 121076 & 87072 & 6835 \\
Media del \% endogamia* & 0,49 & 0,55 & 0,57 \\
Mínimo (\%) & 0,0 & 0,0 & 0,0 \\
Máximo (\%) & 46,88 & 37,50 & 30,62 \\
Endogamia a diferentes niveles de riesgo (IC 95\%) & $\geq 2,5 \%$ & $22,0(21,6-22,5)$ & $37,4(36,7-38,1)$ & $27,9(25,6-30,2)$ \\
& $\geq 5,0 \%$ & $5,1(4,9-5,4)$ & $10,6(10,1-11,0)$ & $8,9(7,5-10,4)$ \\
& $\geq 6,25 \%$ & $3,9(3,5-4,1)$ & $7,8(7,4-8,2)$ & $7,9(6,6-9,3)$ \\
\hline
\end{tabular}

$\mathrm{N}=$ tamaño de muestra.

*Toma en cuenta los animales sin endogamia (Holstein=93 711, Jersey=69 370, Pardo Suizo=5333).

encontrados por Vargas y Gamboa (2008) de $1,25 \%$ y $0,75 \%$ para las mismas razas en hatos de Costa Rica. El promedio general de la población en estudio fue de $\mathrm{F}=0,42 \%$, con valores individuales que oscilan entre 0,0 y $46,88 \%$. Un dato muy similar a este es el descrito por Casas y Tewolde (2001), con $\mathrm{F}=0,44 \%$ en vacas lecheras de hatos costarricenses. Por el contrario, Røkouei et al . $(2010)$ reportan $\mathrm{F}=2,90 \%$ con un rango entre 0,0 y 47,3\%, en una población de vacas Holstein en Irán. Cuando solo se tomó en cuenta animales con $\mathrm{F}>0$, resultó un promedio de $\mathrm{F}=2,36 \%$.

La raza que presentó un mayor $\mathrm{F}$ promedio, entre las vacas con algún porcentaje de endogamia, es la Jersey con $2,68 \%$, seguida de la Holstein con 2,16\% (Cuadro 3). Vargas y Gamboa (2008) también indican que Jersey tiene niveles más elevados de $\mathrm{F}$ que Holstein, esto debido, posiblemente, a que la población de Jersey es más pequeña y, por tanto, hay una menor cantidad disponible de toros de esa raza destinados a inseminación artificial, lo que provoca mayor probabilidad de cruces endogámicos. Sewalem et al. (2006) mencionan valores más elevados, 3,6\% y $3,2 \%$ para las mismas razas en Canadá.

Importante es el hecho de que las razas Jersey y Pardo Suizo presentaron los mayores porcentajes de vacas con niveles de endogamia considerados de riesgo (>6,25\%), con 7,8 (IC 95\%: 7,4-8,2) y 7,9 (IC 95\%: 6,6-9,3), mientras que la Holstein presentó casi la mitad (Cuadro 4).

\section{Efecto absoluto de la endogamia sobre los paráme- tros reproductivos}

La regresión lineal múltiple demuestra que hubo $-0,6 \mathrm{~m}(-18 \mathrm{~d})$ de EPP $(\mathrm{p}<0,001)$ para las vacas con $\mathrm{F}$ bajo $(<6,25 \%)$ con relación a las que tienen niveles de $\mathrm{F}$ elevados. Otros estudios en vacas lecheras de Costa Rica (Casas y Tewolde 2001) y en Estados Unidos de América (Smith et al. 1998), reportan datos diferentes; $+0,5 \mathrm{y}+0,55 \mathrm{~d}$ de EPP por cada $1 \%$ de consanguinidad. Este aspecto es muy importante considerarlo cuando se evalúan las implicaciones económicas de la consanguinidad, ya que cada día que un animal no produce representa un gasto adicional para el productor, lo que disminuye la rentabilidad de la empresa lechera. Se encontró +1,64 m de EPP en Holstein que en Jersey, que es esperable para esta raza, ya que tiende a ser menos precoz que la Jersey. También hubo un impacto significativo de las ecozonas sobre esta misma variable a excepción del bh-P. Las vacas que estaban en el bh-T tienen $+2,5 \mathrm{~m}(\mathrm{p}<0,001)$ de EPP que las que habitaban en la zona ecológica bp-MB (Cuadro 5). 
Cuadro 5. Efecto de endogamia $\geq 6,25 \%$, época de parto, razas y ecozonas sobre edad al primer parto (meses), en vacas Holstein y Jersey de fincas lecheras especializadas de Costa Rica. Período enero 1995 a diciembre 2010.

\begin{tabular}{lcccc}
\hline Variable & Estrato & Diferencia & Error estándar & P \\
\hline Intercepto & 0 & 26,83 & 0,14 & $<, 0001$ \\
Endogamia & $\geq 6,25 \%$ & 0 & $\cdot$ & $\cdot$ \\
\multirow{3}{*}{ Razas } & $<6,25 \%$ & $-0,64$ & 0,12 & $<, 0001$ \\
& Jersey & 0 & $\cdot$ & $\cdot$ \\
Ecozonas & Holstein & 1,63 & 0,06 & $<, 0001$ \\
& bp-MB & 0 & $\cdot$ & $\cdot$ \\
& bh-MB & 0,71 & 0,10 & $<, 0001$ \\
& bh-P & 0,15 & 0,09 & 0,1157 \\
& bh-T & 2,54 & 0,33 & $<, 0001$ \\
& bmh-MB & 2,10 & 0,12 & $<, 0001$ \\
& bmh-P & 0,58 & 0,09 & $<, 0001$ \\
& bmh-T & 1,65 & 0,10 & $<, 0001$ \\
Época de nacimiento & Lluviosa & 0 & $\cdot$ & $\cdot$ \\
& Seca & $-0,18$ & 0,06 & 0,0012 \\
\hline
\end{tabular}

bp-MB= bosque pluvial montano bajo; bh-MB= bosque húmedo montano bajo; bh- $\mathrm{P}=$ bosque húmedo premontano; bh-T= bosque húmedo tropical; bmh- $\mathrm{MB}=$ bosque muy húmedo montano bajo; bmh- $\mathrm{P}=$ bosque muy húmedo premontano; bmh- $\mathrm{T}=$ bosque muy húmedo tropical.

En DA resultó $+0,53$ d para animales con endogamia baja con relación a la elevada, pero no fue significativo. Este es un valor diferente al reportado por Røkouei et al. (2010), que mencionan $+0,53 \mathrm{~d}$ abiertos por cada $1 \%$ de endogamia. Además, se puede notar que la época de parto y número de lactancias tienen efecto, pero solamente son importantes a partir de la segunda lactancia. Todas las zonas ecológicas tuvieron influencia sobre esta variable. Los animales que viven en la ecozona Bh-t tienen más DA y los del Bh-p los que tienen menos en relación a las demás $(p<0,001)$. La raza Holstein tuvo $+31,0 \mathrm{~d}$ abiertos que la Jersey $(\mathrm{p}<0,005)$ (Cuadro 6).

Para S/C hubo $+0,08(\mathrm{p}<0,001)$ servicios en vacas con endogamia baja sobre las que tienen $\mathrm{F}$ alto. La época de parto y la ecozona Bh-t no tuvieron efecto importante sobre esta variable. La raza Holstein tuvo $+0,15 \mathrm{~S} / \mathrm{C}$ que la Jersey (Cuadro 7). Para esta variable no se encontró literatura con la que se pudiesen contrastar los resultados. Las vacas de la primera lactancia mostraron menos $\mathrm{S} / \mathrm{C}$ que los animales de las demás lactancias $(\mathrm{p}<0,001)$.

\section{Riesgo de efectos negativos de endogamia $\geq 6,25 \%$ sobre parámetros reproductivos}

Los animales con $\mathrm{F}$ alto tienen 1,23 veces el riesgo de presentar EPP mayor a $28 \mathrm{~m}$, que los de endogamia baja $(p<0,05)$. Este resultado es consistente con lo encontrado en el modelo lineal, que indicó más de EPP para las vacas con $\mathrm{F}$ alto. Las de raza Holstein tienen 2,12 veces la probabilidad de tener EPP mayor a $28 \mathrm{~m}$ que las Jersey, dato que también corresponde con el obtenido en el modelo lineal con $+1,64 \mathrm{~m}$ en Holstein para esta variable. También hay influencia significativa de la mayoría de las ecozonas, es importante recalcar que las vacas de las zonas ecológicas que tienen más 
Cuadro 6. Efecto de endogamia $\geq 6,25 \%$, época de parto, razas y ecozona sobre días abiertos, en vacas Holstein y Jersey de fincas lecheras especializadas de Costa Rica. Período enero 1995 a diciembre 2010 .

\begin{tabular}{|c|c|c|c|c|}
\hline Variable & Estrato & Diferencia & $\begin{array}{c}\text { Error } \\
\text { estándar }\end{array}$ & $\mathbf{P}$ \\
\hline Intercepto & 0 & 116,65 & 1,87 & $<, 0001$ \\
\hline \multirow[t]{2}{*}{ Endogamia } & $\geq 6,25 \%$ & 0 & . & . \\
\hline & $<6,25 \%$ & 0,53 & 1,40 & 0,7061 \\
\hline \multirow[t]{2}{*}{ Razas } & Jersey & 0 & . & . \\
\hline & Holstein & 31,00 & 0,72 & $<, 0001$ \\
\hline \multirow[t]{7}{*}{ Ecozona } & bp-MB & 0 & . & . \\
\hline & bh-MB & 4,40 & 1,22 & 0,0003 \\
\hline & bh-P & $-6,84$ & 1,13 & $<, 0001$ \\
\hline & bh-T & 25,49 & 4,13 & $<, 0001$ \\
\hline & bmh-MB & 3,73 & 1,48 & 0,0114 \\
\hline & bmh-P & 12,96 & 1,14 & $<, 0001$ \\
\hline & bmh-T & 18,42 & 1,20 & $<, 0001$ \\
\hline \multirow[t]{5}{*}{ Lactancias } & $\geq 5^{\circ}$ & 0 & . & . \\
\hline & $1^{\circ}$ & $-0,31$ & 1,12 & 0,7821 \\
\hline & $2^{\circ}$ & $-3,20$ & 1,17 & 0,0064 \\
\hline & $3^{\circ}$ & $-4,46$ & 1,26 & 0,0004 \\
\hline & $4^{\circ}$ & $-4,52$ & 1,39 & 0,0012 \\
\hline \multirow[t]{2}{*}{ Época de parto } & Lluviosa & 0 & . & . \\
\hline & Seca & $-0,89$ & 0,68 & 0,1915 \\
\hline
\end{tabular}

$\mathrm{bp}-\mathrm{MB}=$ bosque pluvial montano bajo; bh- $\mathrm{MB}=$ bosque húmedo montano bajo; bh- $\mathrm{P}=$ bosque húmedo premontano; bh- $\mathrm{T}=$ bosque húmedo tropical; bmh-MB= bosque muy húmedo montano bajo; bmh- $\mathrm{P}=$ bosque muy húmedo premontano; bmh- $\mathrm{T}=$ bosque muy húmedo tropical.

probabilidad de tener mayor EPP es la de bh-T $(\mathrm{OR}=$ 2,65; IC95\% 1,96-3,59), lo que confirma el resultado del modelo lineal (Cuadro 8).

Las vacas con endogamia alta tienen el mismo riesgo de tener DA mayores de $90 \mathrm{~d}(\mathrm{OR}=1,01 ; \mathrm{IC} 95 \%$ 0,95-1,08), no se encontró literatura similar para compararla con esta variable, pero se debe posiblemente a que las variables reproductivas son poco heredables y dependen más del medio. Este resultado coincide con el modelo lineal, en que el efecto de la endogamia y
Cuadro 7. Efecto de endogamia $\geq 6,25 \%$, época de parto, razas y ecozona sobre servicios por concepción, en vacas Holstein y Jersey de fincas lecheras especializadas de Costa Rica. Período enero 1995 a diciembre 2010.

\begin{tabular}{|c|c|c|c|c|}
\hline Variable & Estrato & Diferencia & $\begin{array}{c}\text { Error } \\
\text { estándar }\end{array}$ & $\mathbf{P}$ \\
\hline Intercepto & 0 & 1,82 & 0,03 & $<, 0001$ \\
\hline \multirow[t]{2}{*}{ Endogamia } & $\geq 6,25 \%$ & 0 & . & . \\
\hline & $<6,25 \%$ & 0,08 & 0,02 & 0,0004 \\
\hline \multirow[t]{2}{*}{ Razas } & Jersey & 0 & . & . \\
\hline & Holstein & 0,15 & 0,01 & $<, 0001$ \\
\hline \multirow[t]{7}{*}{ Ecozona } & bp-MB & 0 & . & . \\
\hline & bh-MB & 0,13 & 0,02 & $<, 0001$ \\
\hline & bh-P & $-0,04$ & 0,02 & 0,0594 \\
\hline & bh-T & 0,12 & 0,07 & 0,0753 \\
\hline & bmh-MB & 0,05 & 0,02 & 0,0504 \\
\hline & bmh-P & 0,19 & 0,02 & $<, 0001$ \\
\hline & bmh-T & 0,25 & 0,02 & $<, 0001$ \\
\hline \multirow[t]{5}{*}{ Lactancias } & $\geq 5^{\circ}$ & 0 & . & . \\
\hline & $1^{\circ}$ & $-0,11$ & 0,02 & $<, 0001$ \\
\hline & $2^{\circ}$ & $-0,10$ & 0,02 & $<, 0001$ \\
\hline & $3^{\circ}$ & $-0,06$ & 0,02 & 0,0028 \\
\hline & $4^{\circ}$ & $-0,03$ & 0,02 & 0,1636 \\
\hline \multirow{2}{*}{$\begin{array}{l}\text { Época de } \\
\text { parto }\end{array}$} & Lluviosa & 0 & . & $\cdot$ \\
\hline & Seca & 0,02 & 0,01 & 0,1257 \\
\hline
\end{tabular}

bp- $\mathrm{MB}=$ bosque pluvial montano bajo; bh- $\mathrm{MB}=$ bosque húmedo montano bajo; bh-P=bosque húmedo premontano; bh- $\mathrm{T}=$ bosque húmedo tropical; bmh-MB= bosque muy húmedo montano bajo; bmh-P= bosque muy húmedo premontano; bmh- $\mathrm{T}=$ bosque muy húmedo tropical.

de casi todas las variables independientes sobre DA es bajo. Es de esperar que Holstein tenga 2,17 veces el riesgo de tener más DA que Jersey (Cuadro 9).

El Cuadro 10 muestra que las vacas con endogamia baja tienen casi la misma probabilidad de tener S/C elevados, que las de endogamia alta $(\mathrm{OR}=0,92 ; \mathrm{IC} 95 \%$ $0,86-0,98)$. La mayoría de las variables independientes incluidas en este estudio, al igual que en el de regresión lineal tienen un impacto débil sobre la variable dependiente SPC. 
Cuadro 8. Razón de posibilidades de las diferentes variables en estudio con respecto a edad al primer parto, en vacas Holstein y Jersey de fincas lecheras especializadas de Costa Rica. Período enero 1995 a diciembre 2010.

\begin{tabular}{lcccc}
\hline Variable & Efecto & Razón de posibilidades & Intervalos de confianza (95\%) \\
\hline Endogamia & $\geq 6,25 \%$ vs. $<6,25 \%$ & 1,23 & 1,10 & 1,38 \\
Razas & Holstein vs. Jersey & 2,12 & 2,00 & 2,25 \\
Ecozona & bh-MB vs. bp-MB & 1,34 & 1,22 & 1,48 \\
& bh-P vs. bp- MB & 1,05 & 0,96 & 1,15 \\
& bh-T vs. bp- MB & 2,65 & 1,96 & 3,59 \\
& bmh-MB $v s$. bp- MB & 2,19 & 1,95 & 2,45 \\
& bmh-P $v s$. bp- MB & 1,42 & 1,30 & 1,55 \\
& bmh-T vs. bp- MB & 2,36 & 2,14 & 2,59 \\
Epoca de parto & Lluviosa $v$. seca & 1,19 & 1,16 & 1,22 \\
\hline
\end{tabular}

bp-MB= bosque pluvial montano bajo; bh-MB= bosque húmedo montano bajo; bh-P=bosque húmedo premontano; bh$\mathrm{T}=$ bosque húmedo tropical; bmh- $\mathrm{MB}=$ bosque muy húmedo montano bajo; bmh- $\mathrm{P}=$ bosque muy húmedo premontano; bmh-T= bosque muy húmedo tropical.

Cuadro 9. Razón de posibilidades de las diferentes variables en estudio con respecto a días abiertos, en vacas Holstein y Jersey de fincas lecheras especializadas de Costa Rica. Período enero 1995 a diciembre 2010.

\begin{tabular}{|c|c|c|c|c|}
\hline \multirow{2}{*}{$\begin{array}{l}\text { Variable } \\
\text { Endogamia }\end{array}$} & Efecto & \multirow{2}{*}{$\begin{array}{c}\begin{array}{c}\text { Razón de } \\
\text { posibilidades }\end{array} \\
1,01\end{array}$} & \multicolumn{2}{|c|}{ Intervalos de confianza (95\%) } \\
\hline & $\geq 6,25 \%$ vs. $<6,25 \%$ & & 0,95 & 1,08 \\
\hline Razas & Holstein $v s$. Jersey & 2,17 & 2,10 & 2,25 \\
\hline \multirow[t]{6}{*}{ Ecozona } & bh-MB vs. bp-MB & 1,00 & 0,94 & 1,06 \\
\hline & bh-P vs. bp- MB & 0,79 & 0,75 & 0,84 \\
\hline & bh-T vs. bp- MB & 1,26 & 1,03 & 1,55 \\
\hline & bmh-MB vs. bp- MB & 0,90 & 0,83 & 0,96 \\
\hline & bmh-P vs. bp- MB & 1,08 & 1,02 & 1,15 \\
\hline & bmh-T vs. bp- MB & 1,11 & 1,04 & 1,18 \\
\hline \multirow[t]{4}{*}{ Lactancias } & $1^{\circ}$ vs. $3^{\circ}$ & 0,96 & 0,92 & 1,01 \\
\hline & $2^{\circ}$ vs. $3^{\circ}$ & 0,99 & 0,94 & 1,04 \\
\hline & $4^{\circ}$ vs. $3^{\circ}$ & 1,01 & 0,95 & 1,08 \\
\hline & $\geq 5^{\circ}$ vs. $3^{\circ}$ & 1,17 & 1,10 & 1,24 \\
\hline Época de parto & Lluviosa $v s$. seca & 1,05 & 1,02 & 1,09 \\
\hline
\end{tabular}

bp-MB= bosque pluvial montano bajo; bh-MB= bosque húmedo montano bajo; bh-P=bosque húmedo premontano; bh$\mathrm{T}=$ bosque húmedo tropical; bmh- $\mathrm{MB}=$ bosque muy húmedo montano bajo; bmh- $\mathrm{P}=$ bosque muy húmedo premontano; bmh-T= bosque muy húmedo tropical. 
Cuadro 10. Razón de posibilidades de las diferentes variables en estudio con respecto a servicios por concepción, en vacas Holstein y Jersey de fincas lecheras especializadas de Costa Rica. Período enero 1995 a diciembre 2010.

\begin{tabular}{|c|c|c|c|c|}
\hline \multirow{2}{*}{$\begin{array}{l}\text { Variable } \\
\text { Endogamia }\end{array}$} & Efecto & \multirow{2}{*}{$\begin{array}{c}\begin{array}{c}\text { Razón de } \\
\text { posibilidades }\end{array} \\
0,92\end{array}$} & \multicolumn{2}{|c|}{ Intervalos de confianza $(95 \%)$} \\
\hline & $\geq 6,25 \%$ vs. $<6,25 \%$ & & 0,86 & 0,98 \\
\hline Razas & Holstein $v s$. Jersey & 1,28 & 1,24 & 1,32 \\
\hline \multirow[t]{6}{*}{ Ecozona } & bh-MB vs. bp-MB & 1,01 & 0,96 & 1,07 \\
\hline & bh-P vs. bp- MB & 0,90 & 0,86 & 0,95 \\
\hline & bh-T vs. bp- MB & 1,18 & 0,97 & 1,43 \\
\hline & bmh-MB vs. bp- MB & 0,93 & 0,87 & 0,99 \\
\hline & bmh-P vs. bp- MB & 1,17 & 1,11 & 1,23 \\
\hline & bmh-T vs. bp- MB & 1,15 & 1,09 & 1,22 \\
\hline \multirow[t]{4}{*}{ Lactancias } & $1^{\circ}$ vs. $3^{\circ}$ & 0,94 & 0,90 & 0,98 \\
\hline & $2^{\circ} v s .3^{\circ}$ & 0,98 & 0,93 & 1,02 \\
\hline & $4^{\circ} v s .3^{\circ}$ & 1,01 & 0,96 & 1,08 \\
\hline & $\geq 5^{\circ}$ vs. $3^{\circ}$ & 1,10 & 1,04 & 1,17 \\
\hline Época de parto & Lluviosa $v s$. seca & 0,98 & 0,95 & 1,02 \\
\hline
\end{tabular}

bp-MB= bosque pluvial montano bajo; bh- $\mathrm{MB}=$ bosque húmedo montano bajo; bh- $\mathrm{P}=$ bosque húmedo premontano; bh- $\mathrm{T}=$ bosque húmedo tropical; bmh-MB= bosque muy húmedo montano bajo; bmh- $\mathrm{P}=$ bosque muy húmedo premontano; bmh- $\mathrm{T}=$ bosque muy húmedo tropical.

\section{LITERATURA CITADA}

Adamec, V; Cassell, BG; Smith, EP; Pearson, RE. 2006. Effects of inbreeding in the dam on dystocia and stillbirths in US Holsteins. J. Dairy Sci. 89:307-314.

AIPL (Animal Improvement Programs Laboratory). 2005. Subject: Inbreeding trends (en línea). Consultado 4 sep. 2011. Disponible en http://aipl.arsusda.gov/ dynamic/inbrd/current/kindx.html

Caravaca, FP; Castel, JM; Guzmán, JL; Delgado, M; Mena, Y; Alcalde, MJ; González, P. 2005. Bases de la producción animal. Sevilla, España, RC Impresiones S.C.A. 506 p.

Caraviello, D. 2004a. Cruzamientos en el ganado lechero. Reproducción y genética. No. 610. Instituto Babcock. Comité de Directores del Sistema de la Universidad de Wisconsin (en línea). Consultado 1 sep. 2011. Disponible en http://babcock.wisc.edu/sites/default/ files/documents/productdownload/du_610.es_.pdf

Caraviello, D. 2004b. Inbreeding in dairy cattle. Reproduction and genetics. No. 615. Instituto Babcock. Comité de Directores del Sistema de la Universidad de Wisconsin (en línea). Consultado 02 sep. 2011. Disponible en http://babcock.wisc.edu/sites/default/files/documents/ productdownload/du_615.en_.pdf

Casas, E; Tewolde, A. 2001. Evaluación de características relacionadas con la eficiencia reproductiva de genotipos criollos lecheros en el trópico húmedo. Archivo Latinoamericano de Producción Animal 9(2):63-67.

Florio, J. 2005. Consanguinidad en la ganadería bovina. Manual de Ganadería Doble propósito 10:129-134.

Holdridge, L. 1987. Ecología basada en zonas de vida. San José, Costa Rica, IICA. 219 p.

Hudson, GFS; Van Vleck, LD. 1984. Inbreeding of artificially bred dairy cattle in the northeastern United States. J. Dairy Sci. 67:161-170.

Lazzari, G; Colleoni, S; Duchi, R; Galli, A; Houghton, FD; Galli, C. 2011. Embryonic genotype and inbreeding affect preimplantation development in cattle. Reproduction 141:625-632.

McParland, S; Kearney, F; Rath, M; Berry, D. 2007. Inbreeding effects on milk production, calving performance, fertility, and conformation in Irish Holstein-Friesians. J. Dairy Sci. 90:4411-4419. 
McParland, S; Kearney, F; Berry, D. 2009. Purging of inbreeding depression within the Irish Holstein-Friesian population. Genetics Selection Evolution 41:16 (en línea). Consultado 5 sep. 2011. Disponible en http:// www.gsejournal.org/content/pdf/1297-9686-41-16.pdf

Nicholas, FW. 2003. Introduction to veterinary genetics. 2 ed. United Kingdom, Blackwell Publishing Ltd. Oxford. 282 p.

Noordhuizen,JPTM; Buurman, J. 1984. Veterinary automated management and production control programme for dairy farms (VAMPP), the application of MUMPS for data processing. Veterinary Quarterly 6:62-77.

Røkouei, M; Vaez-Torshizi, R; Moradi-Shahrbabak, M; Sargolzaei, M; Sørensen, AC. 2010. Monitoring inbreeding trends and inbreeding depression for economically important traits of Holstein cattle in Iran. J. Dairy Sci. 93:3294-3302.

Romero, JJ. 2005. Appraisal of the epidemiology of Neospora caninum infection in Costa Rican dairy cattle. Ph.D. Thesis. The Netherlands, Wageningen University. $137 \mathrm{p}$.

Romero, JJ; Rojas, J; Pérez, E. 2007. Relación de la endogamia con la retención de membranas fetales en vacas de hatos lecheros especializados de Costa Rica. Ciencias Veterinarias 24:79:89.

Ruiz-Flores, A; Núñez-Domínguez, R; Ramírez-Valverde, R; Domínguez-Viveros, J; Mendoza-Domínguez, M; Martínez-Cuevas, E. 2006. Niveles y efectos de la consanguinidad en variables de crecimiento $\mathrm{y}$ reproductivas en bovinos Tropicarne y Suizo Europeo. Agrociencia 40:289-301.

SAS. 2009. SAS/STAT ${ }^{\circledR}$ User's guide: Statistics. Version 9.2. 2 ed. (en línea). Consultado 25 ago. 2011. Disponible en http://support.sas.com/documentation/cdl/en/ statug/63033/HTML/default/viewer.htm\#titlepage. htm

Sewalem, A; Kistemaker, GJ; Miglior, F; Van Doormaal, BJ. 2006. Analysis of inbreeding and its relationship with functional longevity in Canadian dairy cattle. J Dairy Sci. 89:2210-6.

Smith, LA; Cassell, BG; Pearson, RE. 1998. The effects of inbreeding on the lifetime performance of dairy cattle. J. Dairy Sci. 81:2729-2737.

USDA (United States Department of Agriculture). 2012. Trend in inbreeding coefficients for Holstein or Red \& White (en línea). Consultado 11 feb. 2013 Disponible en http://aipl.arsusda.gov/eval/summary/inbrd.cfm

Vargas, B; Gamboa, G. 2008. Estimación de tendencias genéticas e interacción genotipo $\mathrm{x}$ ambiente en ganado lechero de Costa Rica. Téc. Pecu. Méx. 46(4):371386.

Vargas, B; Ulloa, J. 2008. Relación entre curvas de crecimiento y parámetros reproductivos en grupos raciales lecheros de distintas zonas agroecológicas de Costa Rica. Livestock Research for Rural Development. 20 (103) (en línea). Consultado 4 septiembre 2011. Disponible en http://www.lrrd.org/ lrrd20/7/varg20103.htm 
